\title{
Dynamic Dataset of Global Population Mental Wellbeing
}

Jennifer J Newson, PhD1* Tara Thiagarajan, PhD1

'Sapien Labs, Arlington, VA, United States of America
* Correspondence:

Dr. Jennifer Newson

jennifer@sapienlabs.org

\section{Abstract}

Mental wellbeing changes dynamically with life circumstance and is a barometer of current societal health and productivity. Using an online assessment tool called the MHQ, the Mental Health Million project collects real-time global data on population mental wellbeing that can be used to guide public health campaigns and policy making, as well as provides a large-scale dynamic dataset for investigative research. Here we describe this dataset which includes 47 individual indicators of mental wellbeing, 30 life context metrics that place people within their personal circumstance, as well as aggregate scores of mental wellbeing overall, and across 6 dimensions. We provide a detailed summary of the MHQ assessment methodology and recruitment outreach strategy that capitalizes on the opportunity afforded by the global growth in internet connectivity. The dataset automatically updates over time and is available for researchers to download for non-commercial use. As of May 2021, there were 104,000 responses in the dataset, spanning 10 English-speaking countries. By the end of 2021, the MHQ will be launched in Spanish and Arabic, facilitating the expansion of the dataset to cover an additional 20 countries. A summary of key trends from 2020 are provided in the Annual Mental State of the World Report 2020. 


\section{Background \& Summary}

Our mental health and wellbeing is central to our health and productivity, and evolves with life circumstance and the wider social, economic and environmental landscape. Disruptions to this landscape, such as brought about by the Covid-19 pandemic (Varma et al., 2020; Aknin et al., 2021), the experience of life traumas and adversities (Turner and Lloyd, 1995; Kessler et al., 2010; McGrath et al., 2017), and environmental changes such as pollution and toxicity (Xue et al., 2019; Dickerson et al., 2020; Ventriglio et al., 2021) can increase the risk for mental illness and its disabling life consequences. At a societal level there is therefore an opportunity, through policy making and healthcare campaigns, to induce largescale shifts in mental health and wellbeing to the benefit of the wider population (Huppert, 2004; Sampson and Galea, 2018; Fuhrer and Keyes, 2019) while at an individual level, more comprehensive profiling can lead to greater precision in treatment selection (Fernandes et al., 2017; Wium-Andersen et al., 2017). To this end, there is a requirement for large-scale, detailed and real-time surveillance data that profiles the mental health and wellbeing of the general population, with a global reach.

Current epidemiological databases, such as the Global Burden of Disease (GBD) dataset (James et al., 2018; Naghavi, 2019), and World Mental Health Survey Initiative (Kessler et al., 2009), typically provide data on the prevalence of clinical disorders, reflecting the proportion of individuals with a diagnosed mental health disorder. However, specific disorder-based epidemiology misses comorbidities which are the norm (Maj, 2005; Plana-Ripoll et al., 2019), and fails to provide a complete mental wellbeing profile that allows for both aggregate mental wellbeing assessment of the general population as well as more precise understanding of the individual.
Furthermore, the current lack of effective treatments for mental health disorders (Tricklebank et al., 2021) and the perceived barriers to help seeking (Galdas et al., 2005; Henderson et al., 2013), means there is a need for more preventative public health approaches which rely on the identification of social determinants that can be affected through policy, investment and interventions, and targeting of at-risk populations who have not yet reached the threshold for clinical diagnosis. Although many governments include questions on mental health and wellbeing in national initiatives such as the Understanding Society survey in the UK (e.g. Ferry et al., 2021) and the Behavioral Risk Factor Surveillance System (BRFSS) in the US (e.g. Strine et al., 2008), and the Covid-19 pandemic has led to an explosion of studies on mental health in the general population (see here for a list of ongoing longitudinal studies), studies are typically limited in either scope, size, coverage and/or duration. The global growth of internet connectivity has unlocked the opportunity for large-scale data collection of population mental health to track trends, predict risks and monitor intervention outcomes over the longer-term, as well as during times of national and international emergency.

The Mental Health Million project was established with the mission to collect global data on the mental wellbeing status of 1 million people annually by 2024. In line with definitions from the WHO (World Health, 2005), mental wellbeing reflects the breadth of our emotional, social and cognitive capability and incorporates the spectrum of functioning, ranging from symptom characteristics of mental health disorders, through to positive assets which allow people to perform optimally in life. In addition, individuals are not isolated from their environment and life situation but instead reactive to it. The project therefore utilizes a tool called the MHQ that assesses individual elements of mental function as well as 
the wider context of the person's life experience, environment,demographics and situation to provide a holistic and comprehensive perspective across multiple data indicators. An annual Mental State of the World report (Newson et al., 2021) describes top-line trends from the year.

Here we present this dynamic dataset that is freely available for non-commercial use. As of May 2021, the database includes the mental wellbeing status of 104,000+ individuals, spanning 169 countries (including 10 countries with 1000+ responses), with data collection beginning in April 2020 and currently ongoing (400-1000 new responses added every day). The dataset incorporates 47 different elements of mental wellbeing and 30 descriptors of life experience and circumstance which together provide a comprehensive overview of populations over time. The data can be used for numerous purposes, including investigating research questions to understand empirical prevalence of mental health symptoms, changes in mental health status over time and social determinants of mental health outcomes that can support public health campaigns and guide policy making.

\section{Methods}

\section{Data Collection}

Database records are compiled from data collected using a freely available, online, anonymous assessment tool called the Mental Health Quotient $(\mathrm{MHO})$ that takes approximately 15 minutes to complete (Newson and Thiagarajan, 2020). Only adults aged over 18 are permitted to take the assessment. The database automatically updates every time someone completes the MHQ assessment.

\section{Description of the MHQ}

The $\mathrm{MHQ}$ was developed based on a comprehensive coding of mental health symptoms across 126 different mental health questionnaires and interviews spanning depression, anxiety, bipolar disorder, attention-deficit/hyperactivity disorder (ADHD), post-traumatic stress disorder (PTSD), obsessivecompulsive disorder (OCD), addiction, schizophrenia, eating disorder, and autism spectrum disorder (ASD), as well as cross-disorder tools (Newson et al., 2020). A total of 10,154 questions were coded and consolidated into a set of 43 symptom categories. The resultant items were then reviewed in the context of other transdiagnostic frameworks including the Research Domain Criteria (RDoC) put forward by the National Institute of Mental Health (Insel et al., 2010) and symptoms relevant to dementia, and reorganized into a set of 47 indicators of mental wellbeing (Table 1). Each indicator is rated using a 9-point life-impact rating scale which is designed depending on whether the indicator exists on a spectrum from positive to negative (spectrum indicators) or as varying degrees of problem severity (problem indicators). For spectrum indicators 1 on the 9-point scale refers to "Is a real challenge and impacts my ability to function effectively," while 9 refers to "It is a real asset to my life and my performance," and 5 refers to "Sometimes I wish it was better, but it's ok" (Figure 1a). For problem indicators, 1 on the 9-point scale refers to "Never causes me any problems," 9 refers to "Has a constant and severe impact on my ability to function effectively," and 5 refers to "Sometimes causes me difficulties or distress but I can manage" (Figure 1b). 
Table 1: 47 Indicators of Mental wellbeing from the MHQ

\section{Spectrum items}

- Adaptability to change

- Self-worth and confidence

- Creativity and problem solving

- Drive and motivation

- Stability and calmness

- Sleep quality

- Self-control and impulsivity

- Ability to learn

- Coordination

- Relationships with others

- Emotional resilience

- Planning and organization

- Physical intimacy

- Speech and language

- Memory

- Social interactions and co-operation

- Decision making and risk-taking

- Curiosity, interest, and enthusiasm

- Energy level

- Emotional control

- Focus and concentration

- Appetite regulation

- Empathy

- Sensory sensitivity

- Self-image

- Outlook and optimism

- Selective attention

\section{Problem items}

- Restlessness and hyperactivity

- Fear and anxiety

- Susceptibility to infection

- Aggression toward others

- Avoidance and withdrawal

- Unwanted, strange, or obsessive thoughts

- Mood swings

- Sense of being detached from reality

- Nightmares

- Addictions

- Anger and irritability

- Suicidal thoughts or intentions

- Experience of pain

- Guilt and blame

- Hallucinations

- Traumatic flashbacks

- Repetitive or compulsive actions

- Feelings of sadness, distress, and hopelessness

- Physical health issues

- Confusion or slowed thinking 
Figure 1a: Spectrum Rating Scale

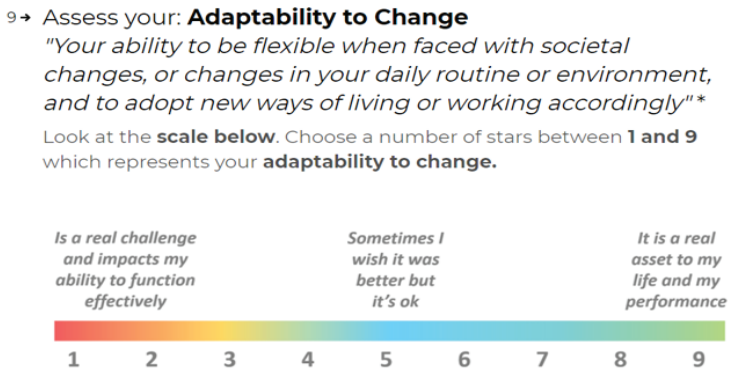

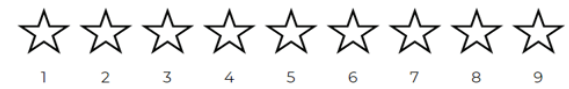

\section{Figure 1b: Problem Rating Scale}

$36 \rightarrow$ Assess your: Restlessness \& Hyperactivity

"The experience of being so fidgety or active that you are unable to relax or be still, even when it is required"*

Look at the scale below. Choose a number between 1 and 9 which represents your restlessness \& hyperactivity.

\begin{tabular}{|c|c|c|c|c|c|c|c|c|}
\hline \multicolumn{2}{|c|}{$\begin{array}{l}\text { Never causes } \\
\text { me any } \\
\text { problems }\end{array}$} & & \multicolumn{3}{|c|}{$\begin{array}{l}\text { Sometimes causes } \\
\text { me difficulties or } \\
\text { distress but I can } \\
\text { manage }\end{array}$} & & \multicolumn{2}{|c|}{$\begin{array}{l}\text { Has a constant and } \\
\text { severe impact on } \\
\text { my ability to } \\
\text { function effectively }\end{array}$} \\
\hline 1 & 2 & 3 & 4 & 5 & 6 & 7 & 8 & 9 \\
\hline 1 & 2 & 3 & 4 & 5 & 6 & 7 & 8 & 9 \\
\hline
\end{tabular}

The MHQ also collects data across 30 data descriptors relating to the demographic, life experience, lifestyle and situational profile of the individual that can be of value in understanding contextual triggers, drivers and determinants of mental wellbeing (Table 2). Demographic descriptors relating to the age, gender, geographical location, employment status, job role, education, household size, race/ethnicity and socioeconomic status of the individual are obtained prior to the collection of mental wellbeing ratings, while life experience (life traumas, Covid-19 impacts, medical conditions, life satisfaction, substance use), lifestyle (sleep, exercise and socializing), help seeking (including reasons for/ not) and situational descriptors (mood, alertness, physical illness, pregnancy) are obtained after the collection of mental wellbeing ratings. Situational elements may be modified from time to time based on the evolving landscape.

\section{Table 2: 30 Contextual descriptors}

Age

Gender

Time of day

Country

State

Zip Code

Race/Ethnicity

Income

Employment

Job Role

Household

Education

How satisfied are you with your life in general?

In general, I get as much sleep as I need:

How regularly to you engage in physical exercise (30 minutes or more)?

How regularly do you socialize with friends in person?

Please select which substances you consume regularly (at least every week)

Do you have a diagnosed medical disorder that significantly impacts your way of life? 
Medical Condition

Are you currently seeking treatment for any mental health concerns?

You answered "No" to the previous question. Please explain further by selecting the following/ What kind of mental health support have you sought/are you currently seeking?

Life Trauma

How would you describe your overall mood right now on a scale from very negative to very positive?

How mentally alert are you feeling right now?

Approximately how many hours did you sleep last night?

Approximately how many hours ago was your last meal?

Physical complaints

Are you currently pregnant?

Covid-health impact

Covid-financial and social impact

The database also records whether the individual found the assessment easy to understand and includes timestamps to reflect the start and completion times for the assessment.

To encourage accurate responses, respondents receive a set of composite MHQ scores (see below) along with tailored feedback on completion of the $\mathrm{MHQ}$ and can opt to receive a more detailed report with recommendations for action via email. For those who request a report via email (on average 49\% of respondents), the email address is converted into an anonymized identifier that can be used to identify repeat respondents within the dataset.

\section{Sample recruitment}

Respondents are recruited through ongoing outreach campaigns operating via Facebook and Google Ads. The Google Ads outreach specifically targets those individuals who are searching for terms relevant to mental health (e.g. psychological test, cognitive assessment test, mental health assessment) with the terms being consistently applied across all countries. Those recruited through this stream may therefore have a specific interest or concern relating to their mental health. Facebook outreach spans individuals who have shown a previous interest in mental health and wellness topics, as well as advertising to all adults in each country with the simple tagline "What is your mental wellbeing score?".

\section{Composite MHQ Scores}

In addition to the raw scores across the 47 spectrum and problem indicators, 7 composite scores are also automatically calculated for each respondent and included in the dataset. These composite scores include an aggregate score over all 47 indicators, as well as for 6 dimensions of mental wellbeing. These scores have multiple purposes. First, they are used to provide easy-to-understand feedback to respondents who receive a report on completion of the $\mathrm{MHQ}$, together with recommendations. In addition, they can be used to provide more aggregated views of the data that can be easier to understand by the general public.

\section{Aggregate MHQ scores}

Aggregate scores position individuals on the spectrum from "Clinical" to "Thriving", spanning a possible range of scores from -100 to +200 where 
negative scores indicate clinical risk. They are not computed as a simple average of raw scores, given (1) there are both negative and positive aspects, (2) there are differences in the seriousness of consequences of different symptom types, and (3) consequences do not necessarily increase linearly at higher values on the scale. Therefore, the raw scores are transformed in 2 steps, which includes a threshold-based rescaling of the 9-point scale to a positive-negative scale, followed by the application of a differential nonlinear weighting of the negative scores to better distinguish at-risk populations. For more details see Newson, 2020 (Newson and Thiagarajan, 2020) and Appendix 1 Mental State of the World Report (Newson et al., 2021).

The resulting rescaled and nonlinearly weighted scores across all problem and spectrum indicators are then summed to provide an aggregate intermediate score. This intermediate score can be either a negative or positive score, where negative scores identify those respondents who had, or were at risk for, a clinical mental health issue and positive scores represent a normal or healthy range of mental health. To compute the MHQ, positive scores are then normalized to a scale between 0 and 200, whereas negative scores are normalized across a smaller window of -1 to -100 . The negative scale was chosen to be smaller to provide a mitigated number to minimize any psychological distress that could be induced by receiving a highly negative score. Thus, the overall MHQ score spans a possible range from -100 to +200 , where negative scores reflect clinical or clinically at-risk populations and positive scores reflect the distribution of the normal healthy population. This score range was also chosen to be similar to the way that IQ scores are computed, where scores are centralized around 100.

\section{MHQ Dimension Scores}

In addition to the aggregate $\mathrm{MHQ}$ scores, $\mathrm{MHQ}$ scores are computed in the dataset for 6 broad dimensions of mental health: Core Cognition, Complex Cognition, Mood \& Outlook, Drive \& Motivation, Social Self, and Mind-Body Connection, that have overlap and parallels with the RDoC principles of domains and constructs (Insel et al., 2010). To compute these dimension scores, a weighted average of items for each dimension is calculated by weighting spectrum or problem indicators core to the dimension as 1 and spectrum or problem indicators secondary to the dimension as 0.5 . This weighting algorithm was developed based on a review of cognitive and neuroscience models of brain functioning. For example, the item Stability and calmness is coded with a primary 1 weighting in the Mood \& Outlook dimension and a secondary 0.5 weighting in the Mind-Body dimension to reflect its dual components of emotion and physiological response, whereas the item Unwanted, strange, or obsessive thoughts is dual coded with a primary weighting in the Core Cognition dimension and a secondary weighting in the Mood \& Outlook dimension to reflect both the cognitive and emotional elements of this item. In this regard, an item could be assigned to 2 different dimensions and occasionally to 3. Overall, each dimension comprised 10 to 24 items. The dimension scores were then normalized to constrain them to a smaller scale than the overall $\mathrm{MHQ}$ to distinguish them from the overall score. Positive scores are normalized to the range of 0 to 100, whereas negative scores are normalized to the range of -1 to -50 .

\section{Geographic Reach of the Dataset}

The MHQ launched in April 2020 in English and although it is open to all respondents globally (the dataset currently spans 169 countries in total), data collection focused on 10 English speaking countries 
(or countries where there is a large English-speaking population) including United States, Canada, United Kingdom, Ireland, Australia, New Zealand, Singapore, South Africa, India and Nigeria. Recruitment initially focused on the United States, Canada, India, United Kingdom and Australia and was later expanded in December 2020 to include South Africa, New Zealand and Singapore. In February 2021, data collection was further expanded to include Ireland and Nigeria.

Each year the MHQ will be launched in additional languages, and the database will be updated with responses in those respective languages. Table 3 outlines the current number of responses and geographical scope of the database, as well as future plans. This table will be updated each year.

Table 3: Overview of existing dataset and planned future expansion in 2021.

\begin{tabular}{|c|c|c|c|}
\hline Launch Date & $\begin{array}{c}\text { Language } \\
\text { of } \mathrm{MHQ}\end{array}$ & Countries with focused outreach & $\begin{array}{l}\text { Total Number } \\
\text { of responses } \\
\text { (Global; May } \\
\text { 2021) }\end{array}$ \\
\hline $\begin{array}{c}\text { April } \\
\text { 2020-ongoing }\end{array}$ & English & $\begin{array}{l}\text { United States, United Kingdom, Canada, } \\
\text { Australia, New Zealand, South Africa, } \\
\text { Singapore, India, Ireland and Nigeria }\end{array}$ & $104,000+$ \\
\hline Planned 2021 & Spanish & $\begin{array}{c}\text { Mexico, Colombia, Argentina, Spain, } \\
\text { Venezuela, Peru, Ecuador, Chile, Guatemala } \\
\text { and Cuba }\end{array}$ & N/A \\
\hline Planned 2021 & Arabic & $\begin{array}{l}\text { Egypt, Algeria, Sudan, Iraq, Morocco, Saudi } \\
\text { Arabia, Yemen, Syria, Tunisia and the United } \\
\text { Arab Emirates. }\end{array}$ & N/A \\
\hline $\begin{array}{c}\text { Planned } \\
\text { 2021/2022 }\end{array}$ & French & $\begin{array}{c}\text { France, Belgium, Switzerland, Canada, } \\
\text { Democratic Republic of Congo, Côte d'Ivoire, } \\
\text { Madagascar, Cameroon, Burkina Faso, } \\
\text { Niger, Mali, Senegal. }\end{array}$ & N/A \\
\hline
\end{tabular}

\section{Dataset Format and Usage}

The Mental Health Million project data is currently freely available to anyone for not-for-profit purposes through our in-house data repository Brainbase where new MHQ completions are automatically appended in real time. Access to the dataset can be requested via our website, where researchers are asked to provide their contact details, a brief description of how they plan to use the data, as well as confirming that it will be used only for not-for-profit purposes. An invitation to the platform is then sent to the individual via email 
through which they can access and download the data, together with supporting documentation. The choice to use an in-house repository, rather than an existing publicly available repository, is to allow the association between the dataset and our $\mathrm{MHQ}$ assessment platform via a backend system that controls the automatic updating of responses, as well to allow future integration with a data exploration and visualization interface that is currently in development.

\section{Data Format}

The data is presently available on Brainbase as a downloadable csv file organized with each individual as a row. Individual fields are described in supplementary table 1. The main file is dynamic and includes all data for the current year. Data is automatically updated every time someone completes the $\mathrm{MHQ}$ assessment. Currently there are 400-1000 new responses being added each day. Each time the dataset is downloaded from Brainbase it will therefore reflect this updating of responses. In addition, separate csv files provide annual datasets from 2020 and pilot data from 2019.

An analysis interface is presently available through the Brainbase platform in beta and allows the slicing of the dataset into subsets for download based on various criteria such as age, gender and country along with simple graphical visualizations. All visualizations at a country level are weighted by the age and gender to reflect the actual demographics of the country based on the country census or latest population estimates.

\section{Validation and Cleaning of the Dataset}

The dataset is based on data and responses provided by respondents self-selected from the general population and no responses are removed or excluded. The accuracy of the data is therefore subject to people's honesty when answering. To strengthen the accuracy of the data, respondents are made aware at the start of the MHQ that they will receive a score and feedback report at the end of the assessment and can opt to receive a more detailed report via email. As the primary motivation of respondents when completing the $\mathrm{MHQ}$ is to gain insight into their own state of mental wellbeing, this reporting system of scores and feedback therefore encourages them to provide honest answers.

The MHQ is freely available online to anyone who wishes to take it. However, there is a possibility that people who are not proficient in the language in which it is taken will attempt to complete it. To try and account for this, we ask a question at the end of the assessment to determine whether the respondent found the MHQ easy to understand "Did you find this assessment easy to understand?" Yes/No. Although we do not automatically remove respondents who answer "No" from the dataset (currently this equates to $2.5 \%$ of the dataset), it is possible to easily remove these respondents from the dataset if required.

\section{Sampling Limitations and Caveats}

Data is collected from respondents recruited via Facebook and Google Ads, as described above. The data from any one country is therefore not necessarily representative of that country's population and should not be considered as such. Demographic representation of age, gender, ethnicities, socioeconomic status and education may not reflect the true demographic profile of a geography and should be accounted for in analysis. However, the sampling has been carried out in a standardized way across all countries, therefore allowing for some country comparisons within this specific dataset. 
Completion of the MHQ requires online access to the internet and therefore is not currently available for completion by those who do not have internet access. Our sister project, the Human Brain Diversity project aims to collect data from non-internet connected individuals, helping to fill this gap in the data.

The recruitment currently prioritizes respondents in English speaking countries (or countries with considerable English-speaking populations) as the MHQ is only currently available in English. Several translations of the MHQ are planned for 2021 and beyond (starting with Spanish, Arabic and French) which will lead to an expansion of the database as the outreach is concurrently expanded to the relevant countries.

Roll out of the MHQ was carried out at different times across countries. This accounts for why the distribution of data varies across different countries over time.

Several updates to the MHQ have been carried out since its launch in April 2020, most recently in January 2021 (see supplementary PDF file). We encourage users to check the supporting documentation on Brainbase which outlines these changes.

\section{References}

Aknin, L., Neve, J.-E.D., Dunn, E., Fancourt, D., Goldberg, E., Helliwell, J., et al. (2021). A Review and Response to the Early Mental Health and Neurological Consequences of the COVID-19 Pandemic. PsyArXiv. doi: https://doi.org/10.31234/osf.io/zw93g. Dickerson, A.S., Wu, A.C., Liew, Z., and Weisskopf, M. (2020). A Scoping Review of Non-Occupational Exposures to Environmental Pollutants and Adult Depression, Anxiety, and Suicide. Curr Environ Health Rep 7(3), 256-271. doi: 10.1007/s40572-02000280-7.
Fernandes, B.S., Williams, L.M., Steiner, J., Leboyer, M., Carvalho, A.F., and Berk, M. (2017). The new field of 'precision psychiatry'. BMC Medicine 15(1), 80. doi: 10.1186/s12916-017-0849-x.

Ferry, F., Bunting, B., Rosato, M., Curran, E., and Leavey, G. (2021). The impact of reduced working on mental health in the early months of the COVID-19 pandemic: Results from the Understanding Society COVID-19 study. J Affect Disord 287, 308-315. doi: 10.1016/j.jad.2021.03.042.

Fuhrer, R., and Keyes, K.M. (2019). Population Mental Health Today: Confronting the Challenge. American Journal of Public Health 109(S3), S149-S149. doi: 10.2105/AJPH.2019.305188.

Galdas, P.M., Cheater, F., and Marshall, P. (2005). Men and health help-seeking behaviour: literature review. J Adv Nurs 49(6), 616 623. doi: 10.1111/j.1365-2648.2004.03331.x.

Henderson, C., Evans-Lacko, S., and Thornicroft, G. (2013). Mental illness stigma, help seeking, and public health programs. American journal of public health 103(5), 777-780. doi: 10.2105/ AJPH.2012.301056.

Huppert, F.A. (2004). "A Population Approach to Positive Psychology: The Potential for Population Interventions to Promote Well-Being and Prevent Disorder," in Positive psychology in practice. (Hoboken, NJ, US: John Wiley \& Sons Inc), 693-709. Insel, T., Cuthbert, B., Garvey, M., Heinssen, R., Pine, D.S., Quinn, K., et al. (2010). Research Domain Criteria (RDoC): Toward a New Classification Framework for Research on Mental Disorders. American Journal of Psychiatry 167(7), 748-751. doi: 10.1176/ appi.ajp.2010.09091379.

James, S.L., Abate, D., Abate, K.H., Abay, S.M., Abbafati, C., Abbasi, N., et al. (2018). Global, regional, and national incidence, prevalence, and years lived with disability for 354 diseases and injuries for 195 countries and territories, 1990\&\#x2013;2017: a systematic analysis for the Global Burden of Disease Study 2017. The Lancet 392(10159), 1789-1858. doi: 10.1016/S01406736(18)32279-7.

Kessler, R.C., Aguilar-Gaxiola, S., Alonso, J., Chatterji, S., Lee, S., and Ustün, T.B. (2009). The WHO World Mental Health (WMH) Surveys. Psychiatrie (Stuttgart, Germany) 6(1), 5-9.

Kessler, R.C., McLaughlin, K.A., Green, J.G., Gruber, M.J., Sampson, N.A., Zaslavsky, A.M., et al. (2010). Childhood adversities and adult psychopathology in the WHO World Mental Health Surveys. The British journal of psychiatry : the journal of mental science 197(5), 378-385. doi: 10.1192/bjp.bp.110.080499. 
Maj, M. (2005). 'Psychiatric comorbidity': an artefact of current diagnostic systems? British Journal of Psychiatry 186(3), 182184. doi: 10.1192/bjp.186.3.182.

McGrath, J.J., McLaughlin, K.A., Saha, S., Aguilar-Gaxiola, S., AlHamzawi, A., Alonso, J., et al. (2017). The association between childhood adversities and subsequent first onset of psychotic experiences: a cross-national analysis of 23998 respondents from 17 countries. Psychol Med 47(7), 1230-1245. doi: 10.1017/ s0033291716003263.

Naghavi, M. (2019). Global, regional, and national burden of suicide mortality 1990 to 2016: systematic analysis for the Global Burden of Disease Study 2016. Bmj 364, 194. doi: 10.1136/ bmj.194.

Newson, J., Pastukh, V., Sukhoi, O., Taylor, J., and Thiagarajan, T. (2021). "Mental State of the World 2020, Mental Health Million project", (ed.) S. Labs.).

Newson, J.J., Hunter, D., and Thiagarajan, T.C. (2020). The Heterogeneity of Mental Health Assessment. Frontiers in Psychiatry 11(76). doi: 10.3389/fpsyt.2020.00076.

Newson, J.J., and Thiagarajan, T.C. (2020). Assessment of Population Well-Being With the Mental Health Quotient (MHQ): Development and Usability Study. JMIR Ment Health 7(7), e17935. doi: 10.2196/17935.

Plana-Ripoll, O., Pedersen, C.B., Holtz, Y., Benros, M.E., Dalsgaard, S., de Jonge, P., et al. (2019). Exploring Comorbidity Within Mental Disorders Among a Danish National Population. JAMA Psychiatry 76(3), 259-270. doi: 10.1001/jamapsychiatry.2018.3658.

Sampson, L., and Galea, S. (2018). An Argument for the Foundations of Population Mental Health. Frontiers in psychiatry 9, 600-600. doi: 10.3389/fpsyt.2018.00600.

Strine, T.W., Mokdad, A.H., Balluz, L.S., Gonzalez, O., Crider, R., Berry, J.T., et al. (2008). Depression and anxiety in the United
States: findings from the 2006 Behavioral Risk Factor Surveillance System. Psychiatr Serv 59(12), 1383-1390. doi: 10.1176/ ps.2008.59.12.1383.

Tricklebank, M.D., Robbins, T.W., Simmons, C., and Wong, E.H.F. (2021). Time to re-engage psychiatric drug discovery by strengthening confidence in preclinical psychopharmacology. Psychopharmacology. doi: 10.1007/s00213-021-05787-X.

Turner, R.J., and Lloyd, D.A. (1995). Lifetime traumas and mental health: the significance of cumulative adversity. $J$ Health Soc Behav 36(4), 360-376.

Varma, P., Junge, M., Meaklim, H., and Jackson, M.L. (2020). Younger people are more vulnerable to stress, anxiety and depression during COVID-19 pandemic: A global cross-sectional survey. Prog Neuropsychopharmacol Biol Psychiatry 109, 110236. doi: 10.1016/j.pnpbp.2020.110236.

Ventriglio, A., Bellomo, A., di Gioia, I., Di Sabatino, D., Favale, D., De Berardis, D., et al. (2021). Environmental pollution and mental health: a narrative review of literature. CNS Spectr 26(1), 51-61. doi: $10.1017 /$ s1092852920001303.

Wium-Andersen, I.K., Vinberg, M., Kessing, L.V., and Mclntyre, R.S. (2017). Personalized medicine in psychiatry. Nordic Journal of Psychiatry 71(1), 12-19. doi: 10.1080/08039488.2016.1216163. World Health, O. (2005). "Promoting mental health : concepts, emerging evidence, practice : a report of the World Health Organization, Department of Mental Health and Substance Abuse in collaboration with the Victorian Health Promotion Foundation and the University of Melbourne". (Geneva: World Health Organization).

Xue, T., Zhu, T., Zheng, Y., and Zhang, Q. (2019). Declines in mental health associated with air pollution and temperature variability in China. Nat Commun 10(1), 2165. doi: 10.1038/s41467-01910196-y. 